\title{
Novel QR Code Image Watermarking using Wavelet Transform
}

\author{
V. Lokeswara Reddy, PhD \\ Associate Professor Department of CSE \\ K.S.R.M. College of \\ Engineering
}

\author{
S. Noha Raj Kumar \\ M. Tech Student Department of CSE \\ K.S.R.M. College of Engineering
}

\begin{abstract}
In this paper, we describe the Digital Image Processing, patent preservation and authentication is more eloquent. To accomplish this, digital dissimilar watermarking techniques are introduced for the salvation. We analyze an algorithm in wavelet domain using wavelet transformation for a digital indomitable watermarking. In this technique secret data is embedded into a Quick Response (QR) Code image. In this, we implant a binary image determined as watermark, embedded into one of the selected wavelet sub bands. QR code is a label of two dimensional barcode. A Barcode is a machine displayable text which is affiliated to a packet and records the pertinent information. A watermark is a litigation of covering digital information in a wave like image, data etc.
\end{abstract}

\section{General Terms}

Digital Image Processing, Robustness etc.,

\section{Keywords}

Watermarking, Quick Response code, Pertinent

\section{INTRODUCTION}

In modern years, data replacement and the transmittal of digital images have become more and more accessible, with the expeditious disclosure of the Internet and digital storage technology. However, these technologies also make it possible for unscrupulous people to duplicate and distribute unauthorized images with low cost, especially images that have high commercial value, such as satellite image of the ground. To relieve tension and pressure from illegal trade in all industries, some experts have directed their attention to illegal trade, digital piracy, and illegal online trading.

The digital watermark is a latest information security and protection technology. The essential goal is to implant the watermark signal that consist of the electronic signature, date, trademark and ingress into the images, audio, video text, and to scent the watermark signal by a specific technology, to conclude the validity of the concession route and prosecute those who violate on the digital works.

This approach improves the unyielding and rough to repulse geometric deformity of the pixels after printing and scanning. Experiments shows that the proposed method is attainable and capable in resisting printing and scanning attacks, and the applicable scope is wide.

\section{PROPOSED SYSTEM}

The obligated system embedded the image into primary image using (N, N) secret sharing scheme. It could resist contaminations like compression, resize and noise addition. The method operates on frequency domain use transformation like Discrete Fourier Transformation (DFT),
Discrete Cosine Transformation (DCT) and Discrete Wavelet Transformation (DWT) etc., In this we propose the blind watermarking technique by using 2-level Discrete Wavelet Transform(DWT) embedded into QR Code image.

\section{QR CODE}

\subsection{QR Code}

QR (Quick Response) Codes are standard with mobile operators as the barcode can store address and URLs. User can feed into the QR Code which can be coded like display message, data. The QR Code specifies data types or assistance for this data by using the pennant. It assures $Q R$ Code procedure which is able to read the barcode precisely. A QR code consists of black and white modules which represent the encoded data.

\subsection{QR Code structure}

QR Code Structure as dispense in the below Fig - 1 which shows the image of the Pixels and describes what a QR Code framework contains [2].

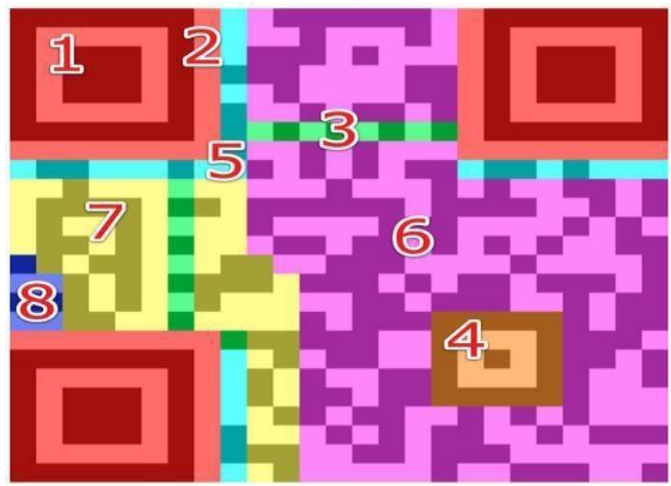

Fig 1: QR Code Structure

Finder Pattern: The three cognate structures that are positioned in the upper corners and in the bottom left corner capacitate the decoder software to recognize. The QR code concludes the correct alignment.

Timing pattern: A sequence of white and black application that refrain from the decoder software to check the width of a single module.

Alignment Pattern: This pattern concedes the QR reader to amend for mutilation when the code is warped or curved. The alignment design emerges on version 2 and higher, consists of alignment designs used depends on the version selected from the encoding.

Format Information: This section consists of 15 bits, error correction rate and the selected mask pattern of the QR code. 
The inaccuracy revision sight can be identified from the two earliest modules of the timing pattern.

Data: Data is concealed into Reed-Solomon-encoded material remnants, it is collected in 8 bit parts (code words) in the data region.

Error Correction: The information code alienation are used in ritual to procreate the error correction (EC) Code words, which are hoard in the error correction region.

In Watermarking Algorithm using 2-Level DWT, disintegrate the watermarked cover image into four sub bands LL, HL, $\mathrm{LH}$, and $\mathrm{HH}$.

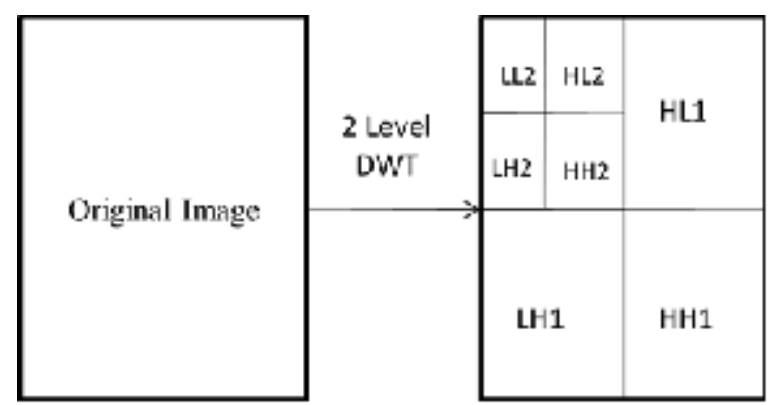

Fig 2: 2- Level Discrete Wavelet Transform

\section{WATERMARKING PROCESS}

In this we can choose a watermark as binary image any data. In this frequency domain we perform the embedding process on QR code image using watermark. As shown in the figure we have to disintegrate the QR code image by two levels using two-dimensional wavelet transformation.

\subsection{Watermark Embedding}

The watermark was produced as a bit sequence of watermark. A prediction of the original value of the pixels was performed using noise elimination technique [3].

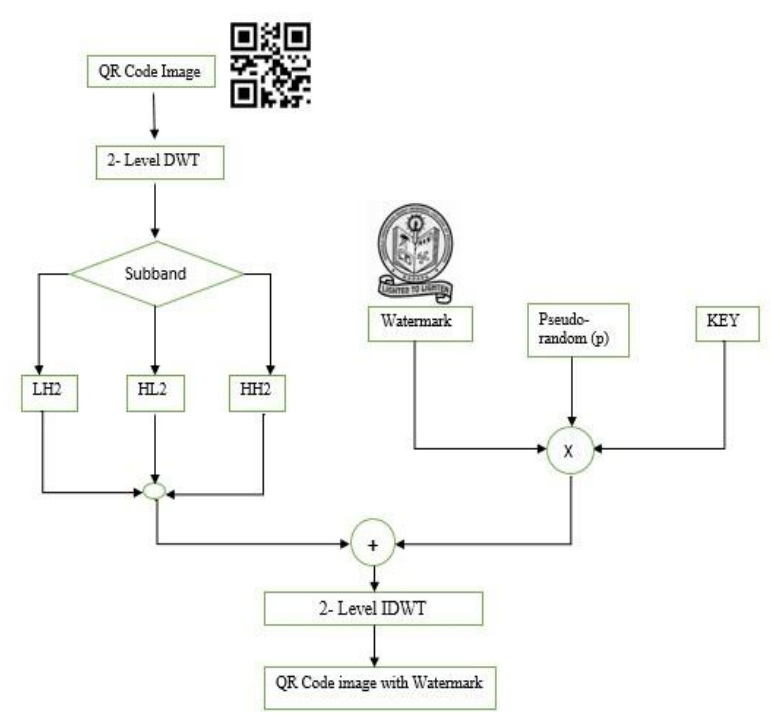

Fig 3: Watermark Embedding Process

The procedure for Watermark embedding is as Follows:

\subsubsection{Steps for Creating watermark image with Secret Key}

Step 1: Run the Application
Step 2: Register as Receiver or Data Owner to Perform the operations in it.

Step 3: Login as Receiver and send a Data request to the Data Owner by clicking on the Data Request button and logout.

Step 4: Login as Data Owner, check the request Status and click on Create Encryption Key.

\subsubsection{Steps for QR code image}

Step 1: Enter the number, click on generate button, the number is converted as image, while saving the image splits into two images and saves as key0 and key1.

Step 2: Then click on Hide Original image with encryption data, select an image, enter the above number, click on embed data and save the File

\section{Step 3: Login as Data Hider}

Step 4: Now we have to encrypt these two key files with two duplicate images, save those Files and logout.

Step 5: Transfer the Encrypt files, Original image to receiver and logout.

\subsection{Watermark Extraction}

The watermark parentage algorithm will not use the primitive $\mathrm{QR}$ code depict. A premonition of the original calibre of the pixels is though essential, a prediction of the original calibre of the pixels was performed using noise elimination technique [3].

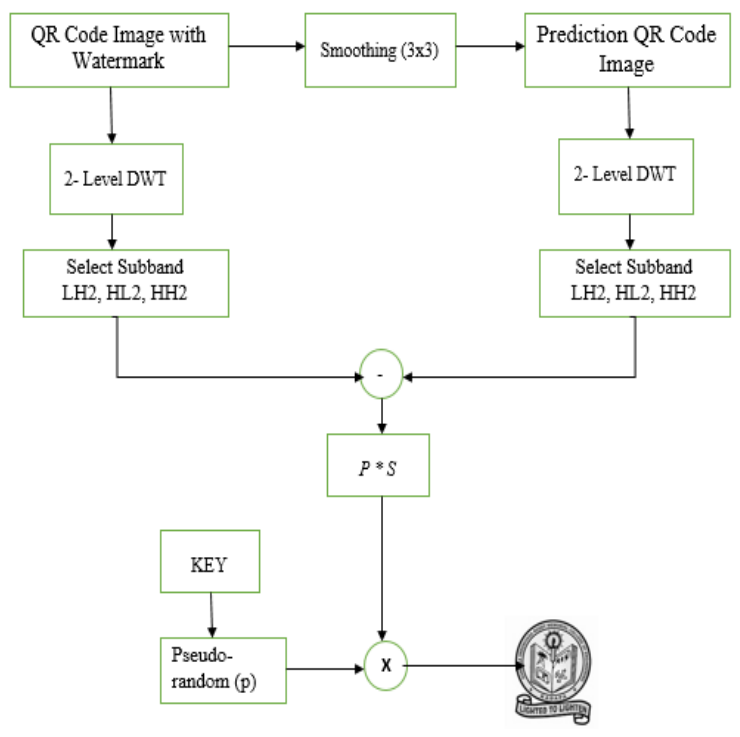

Fig - 4: Watermark Extraction Process

\subsubsection{Steps for Watermark Extraction}

Step 1: Login to Data Hider and click on View Data.

Step 2: Click on Encrypt data with encryption key, decrypt the files, recover the key and logout.

Step 3: Login as receiver, check the status and enter Key to get the Original Image.

Step 4: Close the Application.

As we seen in the above Watermarking Extraction Process, if we don't have the both encrypted files then we can't able to get the Original Image. By using the both decrypted files 
we can extract the key through which only we can get Original Image.

\section{EXPERIMENTAL WORK}

When a System is executed the Process, then it Shows some Results in the form of Snapshots. For the above watermarking process steps the snapshots are here below.

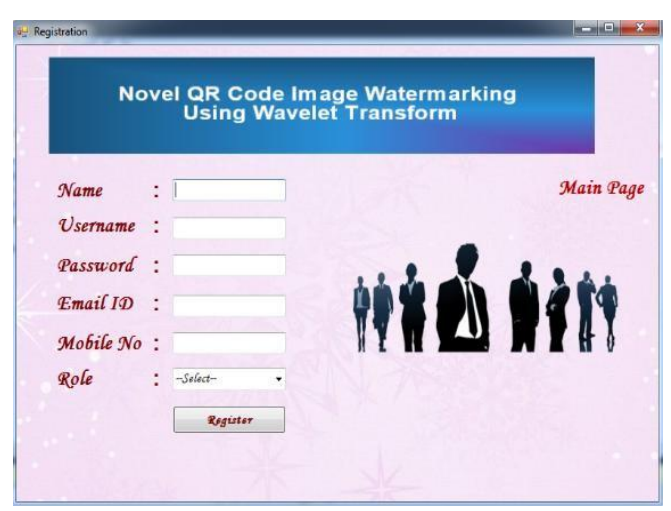

Fig 5: Registration Page

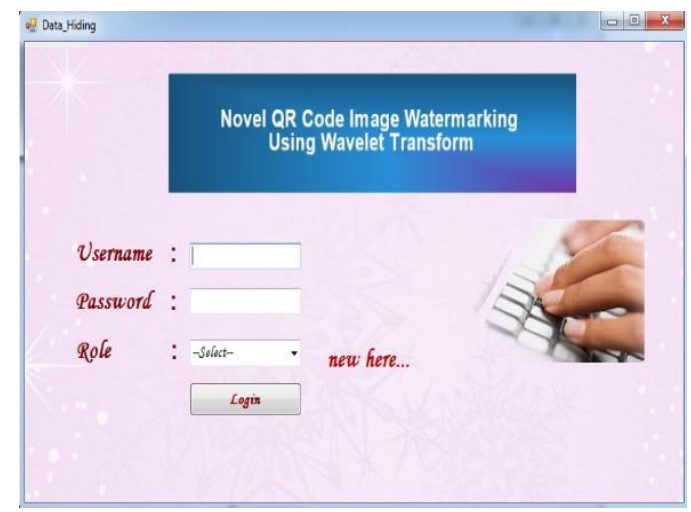

Fig 6: Login Page

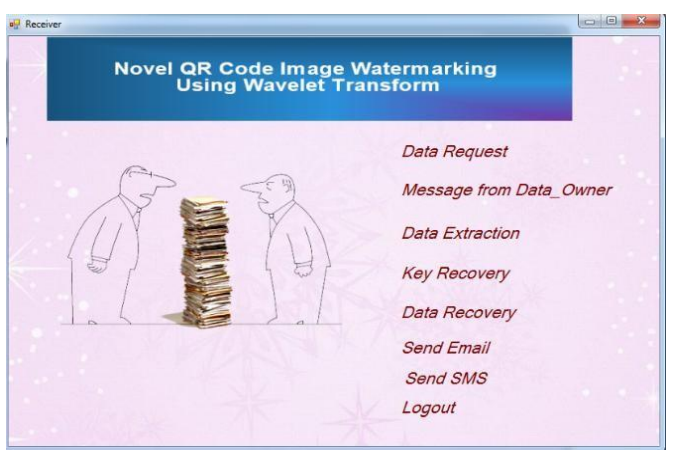

Fig 7: Receiver Page

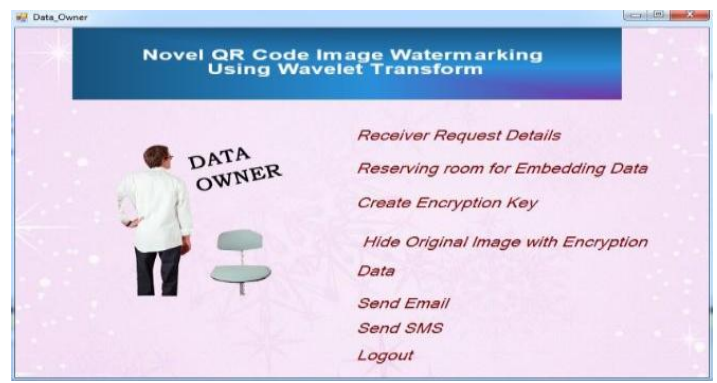

Fig - 8: Data Owner Page

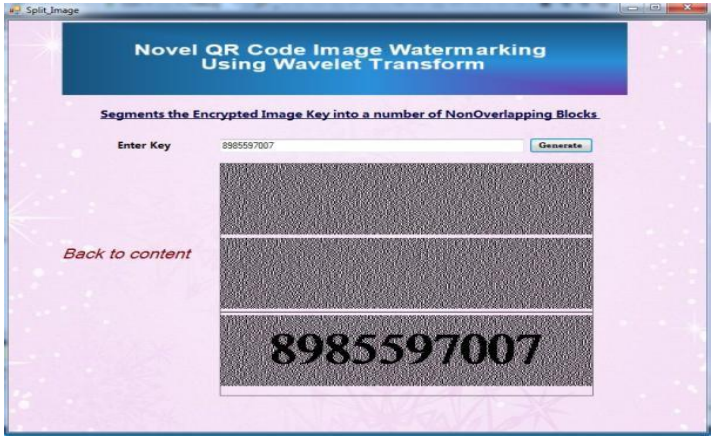

Fig - 9: Generating Key

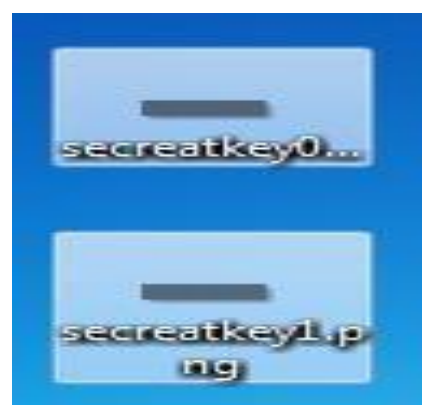

Fig - 10: Secret Key

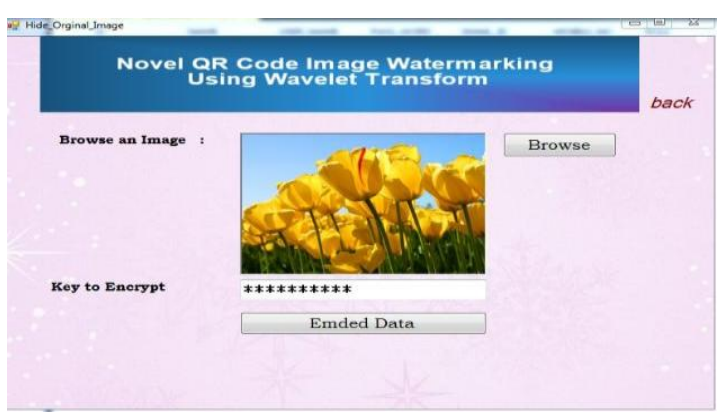

Fig 11: Hiding Original Image with Key

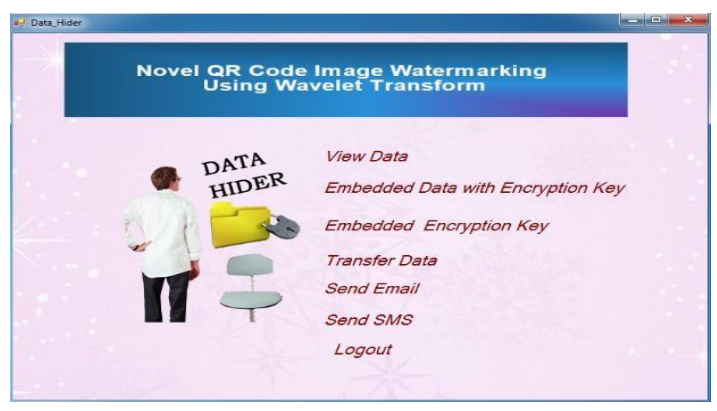

Fig 12: Data Hider Page

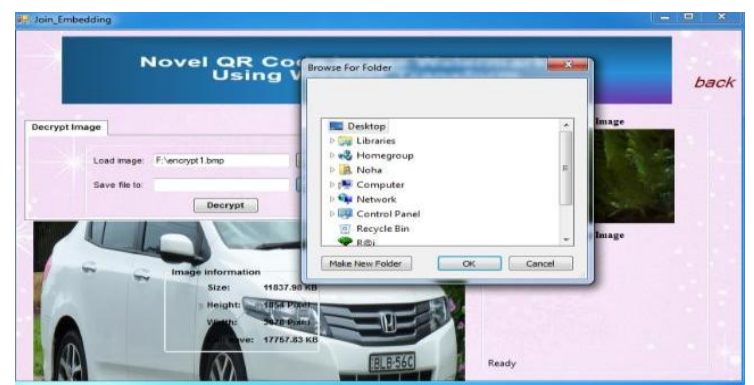

Fig - 13: Decrypting the Image 


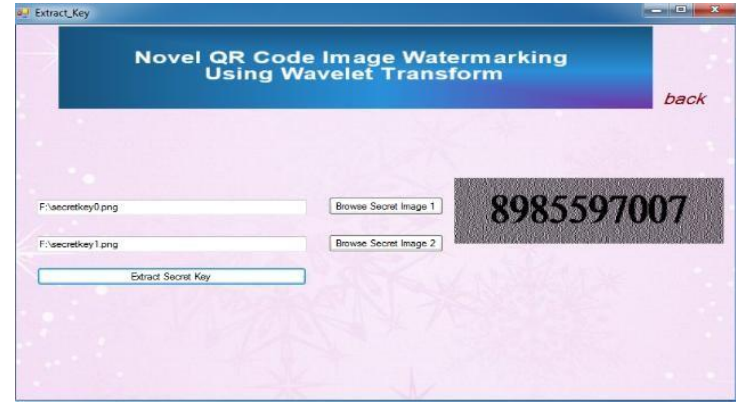

Fig - 14: Extracting Key from the Decrypted Files

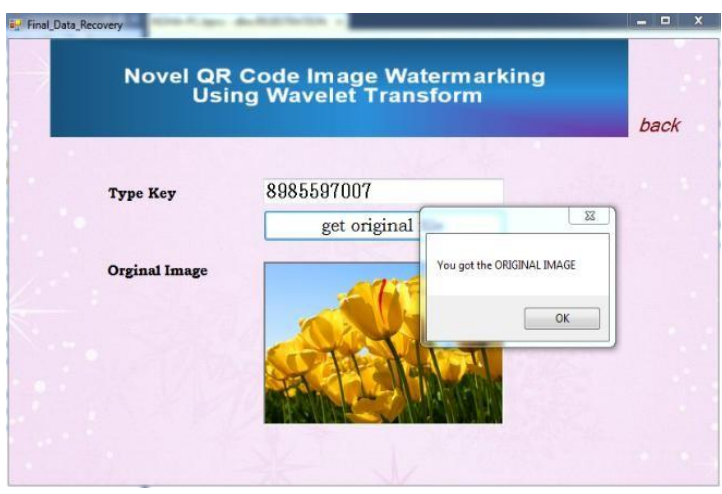

Fig - 15: Original Image

These are the Snapshots of the watermark embedding process and watermark extraction Process.

\section{CONCLUSION}

In the proposed Digital watermarking technique, a binary image is watermarked into a QR Code image. The embedding process is in LH, HL and HH sub bands based on wavelet transform. The algorithm states that the watermark with an acceptable visual quality can be recover easily. In future we try to find more efficient ways for more severe attacks such as stronger noise, high compression and geometric distortion etc.

In future work we focus on extending the proposed method for more embedding capacity and also for embedding secret data in audio or video file. In future there is a scope to develop a better technique for QR Code image based on the above theoretical knowledge and the current technology available and also reduce the degradation of image quality.

\section{REFERENCES}

[1] "Denso wave incorporated," http://www.densowave.com/qrcode/indexe. html.

[2] www.qrcode.meetheed.com AFQjCNHp WeLmnaxeojct 1440228090033515 .

[3] QR Code Watermarking Algorithm based on Wavelet Transform. ISCIT 2013.
[4] Lumini A and Maio D (2000) A wavelet-based image watermarking scheme In:Proc. Int. Conf. Information. Technology: Coding and Computing. pp.122-127.

[5] AlattarAM(2004) Reversible watermark using the difference expansion of a generalized integer transform. IEEE Trans.Image Process. 13: 1147- 1156.

[6] “QRCode,” http://en.wikipedia.org/wiki/QR_code.

[7] Thonky.com. QR Code Tutorial, 2012. http://www.thonky.com/qr-code-tutorial/.Accessed 10 Feb 2013.

[8] Jun-Chou Chuang, Yu-Chen $\mathrm{Hu} \&$ Hsien-Ju Ko. A Novel Secret Sharing Technique using QR Code, International Journal of Image Processing (IJIP), Volume (4) : Issue (5),pp. 468-475, 2010.

[9] Ingemar J. Cox: Digital watermarking and steganography. Morgan Kaufmann, Burlington, MA, USA, 2008

[10] Frank Y. Shih: Digital watermarking and steganography: fundamentals and techniques. Taylor \& Francis, Boca Raton, FL, USA, 2008

[11] A.Z.Tirkel, G.A. Rankin, R.M. Van Schyndel, W.J.Ho, N.R.A.Mee, C.F.Osborne. "Electronic Water Mark". DICTA 93, Macquarie University. p.666-673

[12] Khan, A. and Mirza, A. M. 2007. Genetic perceptual shaping: Utilizing cover image and conceivable attack information during watermark embedding. Inf. Fusion 8 , 4 (Oct. 2007), 354-365

[13] Saraju Mohanty, Nagarajan Ranganathan, and Ravi K. Namballa, VLSI Implementation of Visible Watermarking for a Secure Digital Still Camera Design

\section{AUTHOR PROFILE}

Dr. V. Lokeswara Reddy did his Ph. D in Computer Science and Engineering from JNTUA, Ananthapuramu in the year 2015. He did his M. Tech (CSE) from SRM University, Chennai in the year 2005. He did his M.C.A from S.V. University, Tirupati in the year 2000. He has a total of 13 years of experience in teaching. Currently he is working as Associate Professor at K.S.R.M College of Engineering, Kadapa. He has presented 9 papers in International, National Conferences and published 14 papers in International journals.

S. NOHA RAJ KUMAR did his B. Tech (CSIT) from JNTUA, Ananthapuramu in the year 2012. He is pursuing his M. Tech (CSE) from JNTUA, Ananthapuramu, Andhra Pradesh. He is currently doing his M. Tech in K.S.R.M College of Engineering, Kadapa. 